\title{
Discriminating Neutrino See-Saw Models
}

\author{
M. Hirsch and S. F. King \\ Department of Physics and Astronomy, University of Southampton, Southampton, \\ SO17 1BJ, U.K.
}

\begin{abstract}
We consider how well current theories can predict neutrino mass and mixing parameters, and construct a statistical discriminator which allows us to compare different models to each other. As an example we consider see-saw models based on family symmetry, and single right-handed neutrino dominance, and compare them to each other and to the case of neutrino anarchy with random entries in the neutrino Yukawa and Majorana mass matrices. The predictions depend crucially on the range of the undetermined coefficients over which we scan, and we speculate on how future theories might lead to more precise predictions for the coefficients and hence for neutrino observables. Our results indicate how accurately neutrino masses and mixing angles need to be measured by future experiments in order to discriminate between current models.
\end{abstract}

1 Neutrino oscillation physics is one of the most rapidly developing areas of particle physics [1]. Current atmospheric neutrino data are consistent with $\nu_{\mu} \leftrightarrow \nu_{\tau}$ oscillations [2] with $\sin ^{2} 2 \theta_{23}>0.88$ and $1.5 \times 10^{-3} \mathrm{eV}^{2}<\left|\Delta m_{32}^{2}\right|<$ $5 \times 10^{-3} \mathrm{eV}^{2}\left(90 \%\right.$ CL) [3], where $\Delta m_{i j}^{2}=m_{i}^{2}-m_{j}^{2}$ in units of $\mathrm{eV}^{2}$. CHOOZ data [4] limits $\sin ^{2} 2 \theta_{13}<0.1-0.3$ over the SuperKamiokande (SK) preferred range of $\Delta m_{32}^{2}$. The solar neutrino problem can be solved by four different combinations of oscillation parameters, three of which are based on the Mikheyev-Smirnov-Wolfenstein (MSW) mechanism [5], while the fourth involves neutrino oscillations in vacuum (VAC). These are the well-known large mixing angle (LMA), the small mixing angle (SMA) solutions and a third solution involving a large angle but a lower $\Delta m_{12}^{2}$ (LOW solution). The best-fit points of the various solutions are approximately $\left(\Delta m_{12}^{2}, \sin ^{2} 2 \theta_{12}\right.$, name $)$ [3]: $\left(3.3 \times 10^{-5}, 0.78, \mathrm{LMA} \mathrm{MSW}\right) ;\left(5.1 \times 10^{-6}, 0.0027\right.$, SMA MSW $) ;\left(10^{-7}, 0.93\right.$, LOW MSW $) ;\left(8 \times 10^{-10}, 0.93, \mathrm{VAC}\right)$.

Recently the SNO collaboration [6] has presented its first data on the charged current flux of the solar ${ }^{8} \mathrm{~B}$ neutrinos, which supports both the hypothesis of active neutrino oscillations and the standard solar model [7]. Furthermore results of recent global analyses, performed just days after the SNO result 
[8] show that the most favoured solutions are either the LMA or the LOW solutions, with a slight preference for the LMA solution. The SMA solution is currently disfavoured at about $3 \sigma$, while the VAC regions are disfavoured at various confidence levels.

Because oscillation experiments do not measure absolute values of neutrino masses, in the numerical part of this work we will investigate the following quantities,

$$
\begin{array}{r}
R \equiv\left|\Delta m_{21}^{2}\right| /\left|\Delta m_{32}^{2}\right| \\
s_{C} \equiv 4\left|U_{e 3}\right|^{2}\left(1-\left|U_{e 3}\right|^{2}\right) \\
s_{a t m} \equiv 4\left|U_{\mu 3}\right|^{2}\left(1-\left|U_{\mu 3}\right|^{2}\right) \\
s_{\odot} \equiv 4\left|U_{e 2}\right|^{2}\left|U_{e 1}\right|^{2}
\end{array}
$$

If $U$ is regarded as the lepton mixing matrix, then, in the limit $s_{C} \ll 1$, to very good approximation $s_{C} \approx \sin ^{2} 2 \theta_{13}, s_{a t m} \approx \sin ^{2} 2 \theta_{23}, s_{\odot} \approx \sin ^{2} 2 \theta_{12}$. In the following we will call $\theta_{13}, \theta_{23}, \theta_{12}$ the "CHOOZ", atmospheric, solar angle, although strictly speaking the various experiments measure $s_{C}, s_{a t m}, s_{\odot}$ in the limit $s_{C} \ll 1$. Note that for the LMA MSW solution $R$ is of the order of $R \sim \mathcal{O}\left(10^{-2}\right)$.

Following the experimental confirmation of the atmospheric neutrino problem by the SK collaboration [2], there has been a flood of papers on models of neutrino masses [9]. In order to plan future neutrino experiments it would be very helpful to know how accurate future measurements would need to be to usefully discriminate between the different models, and eventually to decide which (if any) of all these theoretical attempts is the correct one. In order to address this question it is important to understand how well current theories predict neutrino masses and mixing angles. In view of this we find it rather surprising that although hundreds of different models have been proposed there has so far been no attempt to evaluate how well the different models actually predict the neutrino parameters. The main purpose of this paper is to address this issue.

In most of the neutrino mass models [9] based on the seesaw mechanism [10] one usually assumes that there is some underlying broken symmetry which generates a small dimensionless expansion parameter $\lambda$. The entries in the neutrino mass matrix then will depend on $\lambda$ to some - presumably - high power. Such models are called "texture" models, since they produce approximate zeroes in various entries in the mass matrices under consideration (textures). Coefficients in front of the expansion parameter, which are not predicted in these models, are then usually assumed to be order $\mathcal{O}(1)$ numbers, and their influence on the final result is neglected. In this work we are going to investigate the relative importance of the unknown order $\mathcal{O}(1)$ coefficients for the 
first time systematically.

Our basic idea is to construct the following simple neutrino mass model discriminator which we define qualitatively in the following sense: calculate the leading structure (given as powers of $\lambda$ which are fixed by symmetry) of any interesting model and scan "randomly" over the unknown parameters. From these samples one can construct distributions of measurable neutrino parameters, which can be compared to experiment. A model is then considered "good" if the peaks of the distributions coincide with (or are close to) the experimentally preferred value. Such a discriminator is by its nature qualitative rather then quantitative, and it is unclear how much weight should be attributed to a model that predicts all quantities close to the experimentally preferred values. The main problem of the approach is that we have treated all the coefficients on an equal footing, and one can imagine some extra condition which could become known in the future which could single out a particular value for a "random" parameter, which would lead to predictions in the tails of the distributions, rather than near the peaks. However, we would like to argue that although a priori such a possibility cannot be strictly excluded, it might be considered to be contrary to the spirit of texture models.

In our analysis we only consider "high energy scale" models based on the seesaw mechanism [10], which are otherwise hard to test. From the "testability" point of view, low-energy scale models of neutrino mass (see, for example $[11,12])$ might be considered preferable. However, even if one of the low-energy approaches is the true neutrino mass model, a confirmation certainly lies a number of years in the future, and so our methods of discrimination could be useful even in that case.

2 For definiteness we consider [13] models based on a $U(1)$ family symmetry. The idea of such a symmetry is that the three families of leptons are assigned different $U(1)$ charges, and these different charges then control the degree of suppression of the operators responsible for the Yukawa couplings, leading to Yukawa matrices with a hiearchy of entries, and approximate "texture" zeroes. As usual it is assumed that the $U(1)$ is broken by the VEVs of some fields $\theta, \bar{\theta}$ which are singlets under the standard model gauge group, but which have vector-like charges \pm 1 under the $U(1)$ family symmetry. The $U(1)$ breaking scale is set by $\langle\theta\rangle=\langle\bar{\theta}\rangle$. Additional exotic vector matter with mass $M_{V}$ allows an expansion parameter $\lambda$ to be generated by a Froggatt-Nielsen mechanism,

$$
\frac{<\theta>}{M_{V}}=\frac{<\bar{\theta}>}{M_{V}}=\lambda \approx 0.22
$$

where the numerical value of $\lambda$ is motivated by the size of the Cabibbo angle. Small Yukawa couplings are generated effectively from higher dimension non- 
Table 1

\begin{tabular}{||l|r|r|r|r|r|r|r||}
\hline Models & $l_{1}$ & $l_{2}$ & $l_{3}$ & $n_{1}$ & $n_{2}$ & $n_{3}$ & $\sigma$ \\
\hline FC1 & -2 & 0 & 0 & -2 & 1 & 0 & 0 \\
\hline FC2 & -3 & -1 & -1 & -3 & 0 & -1 & 3 \\
\hline FC3 & -1 & 1 & 1 & $\frac{1}{2}$ & 0 & $-\frac{1}{2}$ & -1 \\
\hline FC4 & -1 & 1 & 1 & $\frac{1}{2}$ & $-\frac{1}{2}$ & $-\frac{1}{2}$ & -1 \\
\hline
\end{tabular}

Flavour charges (FC) for four models, as discussed in the text.

renormalisable operators corresponding to insertions of $\theta$ and $\bar{\theta}$ fields and hence to powers of the expansion parameter in Eq.5. The number of powers of the expansion parameter is controlled by the $U(1)$ charge of the particular operator. The lepton doublets, neutrino singlets, Higgs doublet and Higgs singlet relevant to the construction of neutrino mass matrices are assigned $U(1)$ charges $l_{i}, n_{p}, h_{u}=0$ and $\sigma$. From Eq.5, the heavy Majorana matrix elements are $M_{R R}^{i j} \sim A_{i j} \lambda^{\left|n_{i}+n_{j}+\sigma\right|}$. The neutrino Dirac matrix elements are $m_{L R}^{i j} \sim a_{i j} \lambda^{\left|l_{i}+n_{j}\right|}$, where $A_{i j}$ and $a_{i j}$ are undetermined coefficients. For details see $[14]$.

The theory does not determine the coefficients $A_{i j}$ and $a_{i j}$ which we take to be free parameters which are chosen randomly over some range. The physical light effective Majorana neutrino mass matrix which results from the see-saw mechanism [10] is given by $m_{L L}=m_{L R} M_{R R}^{-1} m_{L R}^{T}$, and $m_{L L}$ is diagonalised by a matrix $U$ and the eigenvalues $m_{i}$ are the light neutrino masses. In the basis in which the charged lepton mass matrix is diagonal, $U$ is just the lepton mixing matrix.

In Table 1 we give four examples of models based on different choices of flavour charges. Models FC1-FC3 are examples of single right handed neutrino dominance (SRHND) [14] which naturally lead to small values of $R$, typically $R \sim \lambda^{4}$ (see below). The essential idea of SRHND is that one of the righthanded neutrinos contributes dominantly to the 23 block of $m_{L L}$. This results in the approximate vanishing of the the 23 subdeterminant of $m_{L L}$, and hence one expects a hierarchical spectrum of masses, $m_{\nu_{2}} \ll m_{\nu_{3}}$. Without SRHND a large mixing angle $\theta_{23} \sim \pi / 4$ would typically result in two eigenvalues of the same order of magnitude $m_{2} \sim m_{3}$, and the presence of a mass hierarchy could only be achieved at the expense of some fine-tuning. The amount of fine-tuning required depends on the value of the mass hierarchy.

FC1 and FC3 are taken from [15] where it is shown that they lead to the expectation of a large solar angle. FC1 leads to dominant (from the third righthanded neutrino) and subdominant contributions to $m_{L L}$ [15] (order $\mathcal{O}(1)$ 
coefficients suppressed for simplicity):

$$
m_{L L}^{F C 1} \sim\left(\begin{array}{ccc}
\lambda^{4} & \lambda^{2} & \lambda^{2} \\
\lambda^{2} & 1 & 1 \\
\lambda^{2} & 1 & 1
\end{array}\right)+\mathcal{O}\left(\begin{array}{ccc}
\lambda^{4} & \lambda^{2} & \lambda^{2} \\
\lambda^{2} & \lambda^{2} & \lambda^{2} \\
\lambda^{2} & \lambda^{2} & \lambda^{2}
\end{array}\right) .
$$

We constructed FC2 as an example of a flavour model predicting a small solar angle. FC2 gives dominant and subdominant contributions:

$$
m_{L L}^{F C 2} \sim\left(\begin{array}{ccc}
\lambda^{7} & \lambda^{5} & \lambda^{5} \\
\lambda^{5} & \lambda^{3} & \lambda^{3} \\
\lambda^{5} & \lambda^{3} & \lambda^{3}
\end{array}\right)+\mathcal{O}\left(\begin{array}{ccc}
\lambda^{9} & \lambda^{7} & \lambda^{7} \\
\lambda^{7} & \lambda^{5} & \lambda^{5} \\
\lambda^{7} & \lambda^{5} & \lambda^{5}
\end{array}\right)
$$

FC3 gives a large solar angle, and also a larger CHOOZ angle:

$$
m_{L L}^{F C 3} \sim\left(\begin{array}{ccc}
\lambda & 1 & 1 \\
1 & \lambda^{-1} & \lambda^{-1} \\
1 & \lambda^{-1} & \lambda^{-1}
\end{array}\right)+\mathcal{O}\left(\begin{array}{ccc}
\lambda & \lambda & \lambda \\
\lambda & \lambda & \lambda \\
\lambda & \lambda & \lambda
\end{array}\right) .
$$

The model FC4 is deliberately chosen not to have SRHND, but is designed to give the same leading order structure as $m_{L L}^{F C 3}$, but with subleading contributions to the 23 block which are also of the same order, leading to an unsuppressed value of $R$. We will not consider renormalization group effects here, since it has been shown that they only lead to corrections of several percent [16] which are negligible compared to the effects of the unknown coefficients.

Approximate expectations for the experimentally accessible quantities $\left(\theta_{23}\right.$, $\theta_{13}, \theta_{12}$ and $\left.R \equiv\left|\Delta m_{12}^{2}\right| /\left|\Delta m_{23}^{2}\right|\right)$ may be estimated from the form of the matrices in Eqs.6-8 as discussed in [15] and are given in Table 2.

Finally, as a fifth example we will consider neutrino anarchy [17]. In the language of flavour charges anarchy simply corresponds to choosing $l_{1}=l_{2}=l_{3}$ and $n_{1}=n_{2}=n_{3}$, such that the effective neutrino mass matrix shows no obvious structure. It is therefore completely determined by the random coefficients.

3 As in [17] we investigate the quantities $R, s_{C}, s_{a t m}$ and $s_{\odot}$, as defined in Eqs.1-4. As mentioned in the introduction these are appropriate quantities when considering data from oscillation experiments. Recall, in the limit $s_{C} \ll$ 
Table 2

\begin{tabular}{||l|r|r|r|r||}
\hline Model & $\theta_{23}$ & $\theta_{13}$ & $\theta_{12}$ & $R$ \\
\hline FC1 & 1 & $\lambda^{2}$ & 1 & $\lambda^{4}$ \\
\hline FC2 & 1 & $\lambda^{2}$ & $\lambda^{2}$ & $\lambda^{4}$ \\
\hline FC3 & 1 & $\lambda$ & 1 & $\lambda^{4}$ \\
\hline FC4 & 1 & $\lambda$ & - & - \\
\hline
\end{tabular}

Approximate expectations for $\theta_{23}, \theta_{13}, \theta_{12}$ and $R$ for the four different choices of flavour charges defined in the text.

1, to very good approximation $s_{C} \approx \sin ^{2} 2 \theta_{13}, s_{\text {atm }} \approx \sin ^{2} 2 \theta_{23}, s_{\odot} \approx \sin ^{2} 2 \theta_{12}$. As a test of our numerical procedure we ran a sample calculation with one million randomly chosen points for all five models imposing the same cuts as used in [17] $\left(R<1 / 10, s_{C}<0.15, s_{a t m}>0.5\right.$ and $\left.s_{\odot}>0.5\right)$. For this test we restricted the range of the coefficients to be in the interval $[-1,1]$ in order to be able to compare our calculation to the results presented in [17]. Note, however, that this range is not the appropriate one for texture models, as discussed below, and was used only in this test calculation. We have checked that our results for the anarchy matrix are statistically consistent with the original anarchy calculation in [17], namely that approximately $3 \%$ of the points pass all the cuts. By comparison, with the same procedure applied to model FC1 in Table $125 \%$ of all points pass the cuts.

In texture models, however, it is necessary to choose the range of the coefficients $A_{i j}$ and $a_{i j}$ differently from [-1,1]. As explained in more detail in section 4, where the importance of the coefficients is discussed, in order to not destroy the texture structure imposed by the expansion parameter, we have chosen the range of the coefficients to be $\pm[\sqrt{2 \lambda}, 1 / \sqrt{2 \lambda}]$. In other words the magnitude of each of the coefficients is selected randomly in the range 0.663-1.508 and its sign is also selected randomly. With the structure preserving range only $1.8 \%$ of the neutrino anarchy points pass the cuts, while for the case with structure FC1 56\% of the points now pass the cuts. Structure in the neutrino mass matrix is clearly preferred over anarchy, since more points pass the cut in that case.

In Figure 1 we give distributions for $s_{a t m}, s_{\odot}, s_{C}$ and $R$ for $10^{8}$ points and five different models, using random coefficients in the range $\pm[\sqrt{2 \lambda}, 1 / \sqrt{2 \lambda}]$. Note, that the plots are on a log-linear scale. Using a semilogarithmic scale is suitable for quantities which a priori can be distributed in intervals ranging over several orders of magnitude, as can be expected for the observables we are interested in. Choosing a linear scale a model predicting $s_{\odot}=0.1$, for example, could not be distinguished from a model predicting $s_{\odot}=10^{-3}$. Thus our choice of scale and our definition of a "good" model corresponds to a rather mild requirement. Instead of asking for an acceptance of a given model at some 

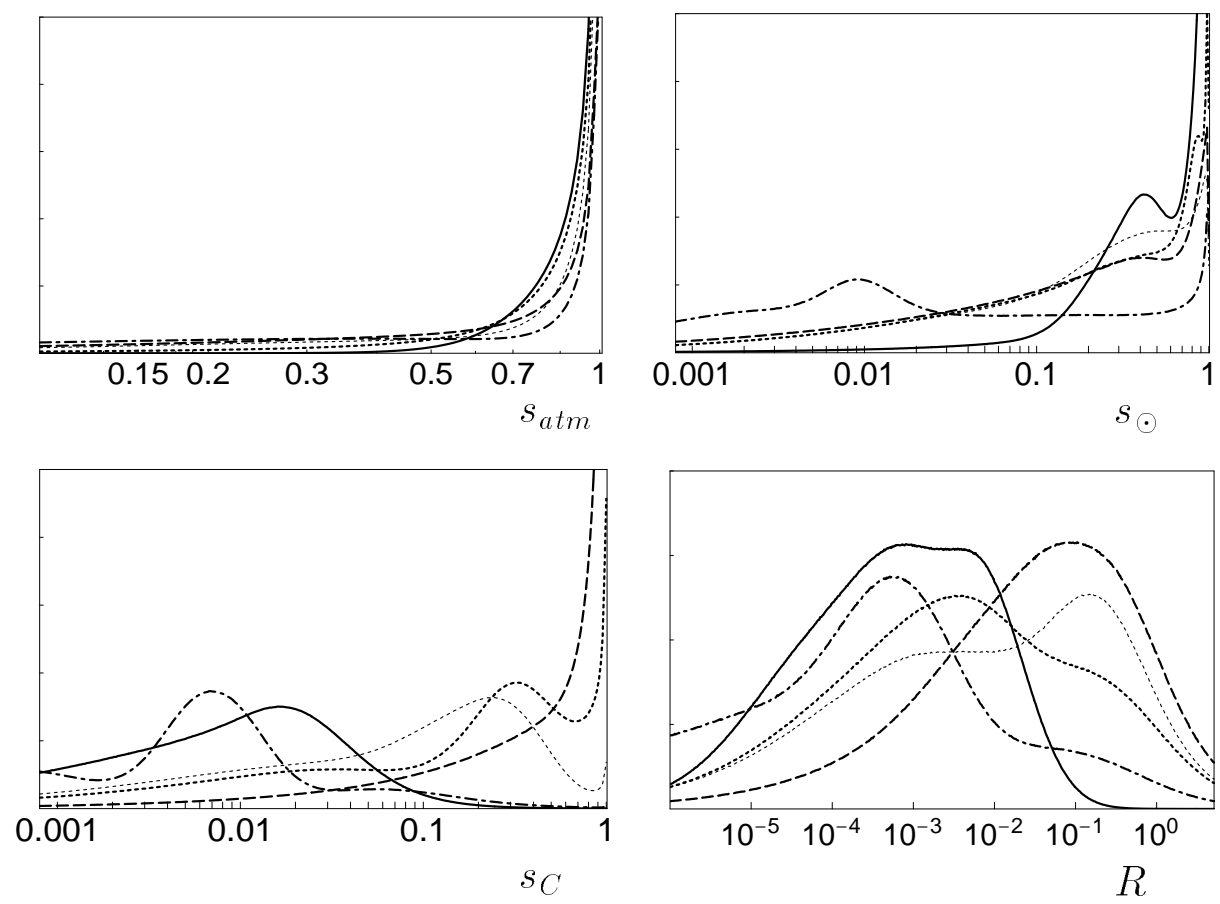

Fig. 1. Theoretical distributions for the predictions of neutrino mass and mixing parameters for five selected see-saw models: FC1 (full), FC2 (dot-dashes), FC3 (thick dots), FC4 (thin dots), anarchy (dashes). Matrix coefficients are randomly chosen in the interval $\pm[\sqrt{2 \lambda}, 1 / \sqrt{2 \lambda}]$. The vertical axis in each panel (deliberately not labelled) represents the logarithmically binned distributions with correct relative normalisation for each model, with heights plotted on a linear scale in arbitrary units.

(high) level of confidence, we ask for the minimum requirement that models should give at least the correct order-of-magnitude estimate for observables. Any model failing even this mild test will fail even more badly once subjected to a more sophisticated numerical analysis.

The dashed line and the full line correspond to the case of neutrino anarchy and the FC1 model as in Table 1. If we focus only on these to begin with we see that they both give a peak at $s_{a t m}=1$, and $s_{\odot}=1$, but the FC1 model gives also a peak at $s_{\odot}=0.4$, and disfavours $s_{\odot}<0.1$.

The main difference between anarchy and FC1 is in the CHOOZ angle where anarchy again gives a peak at $s_{C}=1$, while the FC1 model has a peak near the small value $s_{C}=0.02$. The $\mathrm{R}$ value for the $\mathrm{FC} 1$ model also prefers smaller values than the case of anarchy, albeit with a broader peak, and may be consistent with either the LMA or the LOW solutions. Clearly more accurate experimental determinations of physical parameters will eventually provide a powerful discriminator between the case of neutrino anarchy and neutrino 
structure.

The remaining lines in Figure 1 correspond to the different flavour models FC2, FC3, FC4. All the models predict large values of $s_{\text {atm }}$ and so cannot be distinguished this way. The FC2 model (dot-dash) gives a peak at $s_{\odot}=0.01$, and therefore prefers the SMA MSW solution, consistent with the analytic expectations discussed previously [15]. It also has peaks near $s_{C}=0.01$ and $R=10^{-3}$ consistent with the analytic expectations. The FC3 model (thick dots) is consistent with the LMA or LOW solutions, similar to the FC1 model (solid), since it peaks at large $s_{\odot}$ and the $R$ peak is in the same region, albeit with a different detailed shape. However, unlike FC1, the FC3 model has a peak at the larger value $s_{C}=0.3$ and so may be distinguished by a measurement of $\theta_{13}$. Finally the FC4 model (thin dots) is designed to give the same leading order contributions to $m_{L L}$ as FC3 (thick dots), differing only in the subleading contributions, so that FC4 does not possess the feature of SRHND. This subtle difference between the two models gives rise to a quantitative difference since the peak in $R$ for the FC4 model is shifted to the right compared to that in the FC3 model.

4 We emphasise that in all flavour models of the kind we consider [13] the coefficients $A_{i j}$ and $a_{i j}$ are not specified. In order for these models to make sense it is implicitly assumed that the coefficients are of order unity to within an error determined by the expansion parameter. The range of coefficients applied previously, namely $\pm[\sqrt{2 \lambda}, 1 / \sqrt{2 \lambda}]$, was chosen in order that the structure imposed by the flavour charges and the expansion parameter $\lambda$ was not destroyed. We re-emphasise that this is not guaranteed, but a minimum requirement for texture models in order to make sense physically. We think that future theoretical progress in flavour models of neutrino masses lies in being able to predict these coefficients more accurately, and Figure 2 demonstrates the importance of this point.

In Figure 2 we have plotted the distribution in the solar angle for the case of FC2, but with 3 different ranges of the coefficients. As the range of the coefficients is reduced, the central peak of the distribution becomes more pronounced, with smaller side-peaks emerging as a result of accidental cancellations due to the arbitrary signs of each element which we allow in our Monte Carlo. Thus Figure 2 shows the accuracy of the prediction for the solar angle which may be possible in some future theory where the coefficients are specified more accurately. Analagous results are obtained for the other observables.

5 To conclude, we have considered how well current theories, in particular those based on the see-saw mechanism and SRHND, can predict neutrino mass and mixing parameters, and have proposed a qualitative discriminator based on logarithmic distributions calculated from a random scan over the (presently unknown) coefficients in the neutrino Yukawa matrix and the neu- 


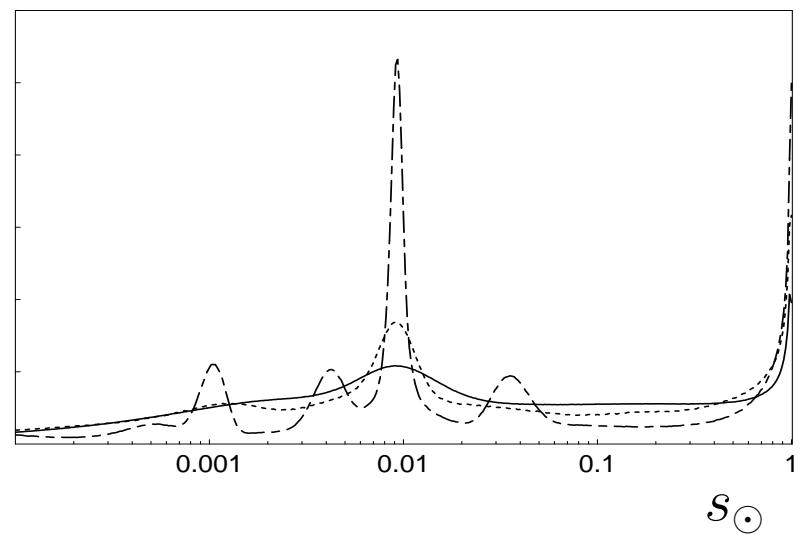

Fig. 2. Plots of $s \odot$ for the case of FC2 with three different ranges of the coefficients $A_{i j}$ and $a_{i j}$. Full line: $\pm[\sqrt{2 \lambda}, 1 / \sqrt{2 \lambda}]$. Dotted line: $\pm[0.82,1.18]$. Dash-dotted line: $\pm[0.95,1.05]$.

trino Majorana matrix, leading to the distributions in Figure 1. This paper is the first serious attempt to show how the uncertainty of the coefficients of the input see-saw matrices feeds through to the uncertainty in the predictions of experimental neutrino observables.

What is the value of such distributions, and what conclusions can be drawn from them? Consider for example the distributions in the CHOOZ angle $s_{C}$ corresponding to the third panel of Figure 1. It is clearly seen that three of the models FC3 (thick dots), FC4 (thin dots) and anarchy (dashes) give distributions which peak at values of $s_{C}$ which are larger than or around the experimental limit $s_{C} \leq 0.1-0.3$. Indeed anarchy peaks at $s_{C}=1$. This panel also shows qualitatively how increasingly unlikely these models will become as the experimental limit on $s_{C}$ becomes stronger, and again anarchy fares the worst having the lowest number of points in the tail of the distribution. We should emphasise that such a plot of the distribution in $s_{C}$ was not shown for anarchy in [17]. On the other hand the models FC1 (full) and FC2 (dot-dash) give distributions which peak at much smaller values of $s_{C}$, in agreement with the analytic estimates in Table 2 . The value of the distributions is that one can see pictorially how much a given experimental limit on $s_{C}$ favours or disfavours a particular model. The conclusions which one draws from such distributions remain a matter of taste, but at least the distributions enable such conclusions to be made based on the best possible information. It is manifestly clear that seeing a probability distribution is more informative than simply having an analytic estimate of the position of the peak of the distribution, and our paper supplies this additional information.

Clearly from the above discussion an accurate measurement of $\theta_{13}$ is very important, since it could rule out or disfavour large classes of models, and this may require a neutrino factory [18]. What about the other neutrino observables? From the first panel in Figure 1 we see that an accurate determination 
of the atmospheric angle $\theta_{23}$ is not so important since all the considered models peak at maximal mixing. However, by contrast, a measurement of the solar angle $\theta_{12}$ is important in resolving large classes of models, and the second panel in Figure 1 shows the distributions in $s_{\odot}$. The recent SNO indication of a large solar angle clearly disfavours the model FC2 (dot-dash) which peaks at a small solar angle according to the analytic estimate in Table 2. One may ask, what do we learn from the distributions that we do not already know from the analytic estimates of the position of the peak? The answer is that the distributions give us a rough idea of the uncertainties of the analytic estimates.

The distributions in $R$, corresponding to the fourth panel in Figure 1, all have rather broad distributions which could not have been predicted by the analytic results, which again only predict the position of the peaks. One would expect that a small measured value of $R$ would favour SRHND over other models where $R$ is larger. This effect is clearly seen since the FC4 models (thin dots) and the anarchy model (dashes) do not have SRHND and therefore give distributions in $R$ in the fourth panel in Figure 1 which are peaked towards larger values than the SRHND models FC1-FC3. However what could not have been predicted is the width of the distributions which are so broad as to make these distributions overlap over large regions of $R$. Once again the distributions provide this additional information which has hitherto been missing from the literature. Moreover, as seen from Table 2, for the models FC1-FC3 one expects $R$ to be $\sim \lambda^{4}$ in all cases, whereas Fig. 1 demonstrates that even the position of the peaks in this variable can vary up to an order of magnitude. Again, the distributions give us an estimate of the limitations of the analytic approaches.

We hope that the additional information provided by the distributions will be of use to our experimental colleagues in planning the next generation of neutrino experiments, but we would caution that future theories could predict smaller ranges of the unknown coefficients, which could demand higher experimental accuracy in the measurement of neutrino mass and mixing parameters than that deduced from the results presented here. Also we emphasise that we have treated all the coefficients as independent and uncorrelated, and some future theory could relate these coefficients in such a way as to give rise to predictions in the tails of the distributions rather than near the peaks. To some extent this undermines the conclusions which we can draw from our current analysis. However we feel that the distributions which we present are a fair reflection of current theoretical uncertainties.

S.K. is grateful to PPARC for the support of a Senior Fellowship. M.H. would like to thank E. Nardi for discussions and the Universidad de Antioquia for the kind hospitality during the early stages of this work. We also would like to thank J. Parry and D. Crooks for a careful reading of the manuscript. 


\section{References}

[1] See, for example, talks at XIX. Int. Conf. on Neutrino Physics \& Astrophysics (Neutrino 2000), http://nu2000.sno.laurentian.ca/; A summary of links on pages related to neutrino oscillations can be found at http://www.hep.anl.gov/ndk/hypertext/nuindustry.html

[2] Y. Fukuda et al. [Super-Kamiokande Collaboration], Phys. Rev. Lett. 81, 1562 (1998).

[3] See e.g. M.C. Gonzalez-Garcia et al., Phys.Rev. D63 (2001) 033005; M.C. Gonzalez-Garcia and C. Peña-Garay, Nucl. Phys. Proc. Suppl. 91 (2000) 80 hep-ph/0009041

[4] M. Apollonio et al., Phys.Lett. B466 (1999) 415.

[5] L. Wolfenstein, Phys. Rev. D17 (1978) 2369; S. Mikheyev and A. Yu. Smirnov, Sov. J. Nucl. Phys. 42 (1985) 913.

[6] Q. R. Ahmad et al. [SNO Collaboration], nucl-ex/0106015.

[7] J. N. Bahcall, M. H. Pinsonneault and S. Basu, ApJ 555 (2001) 990 astro$\mathrm{ph} / 0010346$

[8] G. L. Fogli, E. Lisi, D. Montanino and A. Palazzo, hep-ph/0106247; John N. Bahcall, M. C. Gonzalez-Garcia, Carlos Pena-Garay, hep-ph/0106258.

[9] For a non-exhaustive list of recent reviews, see for example: G. Altarelli and F. Feruglio, Phys. Rep. 320 (1999) 295; R. N. Mohapatra, hepph/0008232; J.W.F. Valle, hep-ph/9911224; S.M. Bilenky, C. Giunti, W. Grimus, Prog.Part.Nucl.Phys. 43 (1999) 1-86; S.F. King, hep-ph/0105261.

[10] M. Gell-Mann, P. Ramond and R. Slansky in Sanibel Talk, CALT-68-709, Feb 1979, and in Supergravity (North Holland, Amsterdam 1979); T. Yanagida in Proc. of the Workshop on Unified Theory and Baryon Number of the Universe, KEK, Japan, 1979.

[11] W. Porod et al., Phys. Rev. D63 (2001) 115004

[12] E. Ma et al., hep-ph/0012101

[13] C. D. Froggatt and H. B. Nilsen, Nucl. Phys. B147 (1979) 277; L. Ibanez and G.G. Ross, Phys. Lett. B332 (1994) 100; P. Binetruy and P. Ramond, Phys. Lett. B350 (1995) 49.

[14] S.F. King, Nucl. Phys. B562 (1999) 57.

[15] S.F. King, Nucl. Phys. B576 (2000) 85.

[16] S.F. King and N.N. Singh, Nucl. Phys. B591 (2000) 3

[17] L. Hall, H. Murayama and N. Weiner, Phys. Rev. Lett. 84, 2572 (2000) hep$\mathrm{ph} / 9911341$.

[18] C.Albright et al, hep-ex/0008064. 